\title{
Evaluation of an immunodot blot technique for the detection of antibodies against Taenia solium larval antigens
}

\author{
Fernando Salazar-Anton • Aleyda Tellez $•$ Johan Lindh
}

Received: 28 September 2011 / Accepted: 30 November 2011 /Published online: 4 January 2012

(C) The Author(s) 2011. This article is published with open access at Springerlink.com

\begin{abstract}
Immunodiagnostic tests represent an important tool for diagnosis of cysticercosis, the disease caused by cysticerci of Taenia solium. Accurate diagnosis of neurocysticercosis (NCC) requires costly neuroimaging techniques (magnetic resonance imaging and computed tomography), which are seldom affordable for people in endemic countries. Hence, new low-cost diagnostic methods offering good sensitivity and specificity are needed. Here, we studied four immunodiagnostic tests immunodot blot Tsol-p27, a commercial ELISA, and Western blot Tsol-p27/TsolHSP36, and compared them with a commercial enzyme-linked immunoelectrotransfer blot (EITB) that we regarded as the gold standard method. The analyzed serum samples were obtained from 160 patients: 94 epileptics suspected of NCC, six individuals confirmed NCC-positive, and 60 with positive (30) or negative (30)
\end{abstract}

Electronic supplementary material The online version of this article (doi:10.1007/s00436-011-2747-z) contains supplementary material, which is available to authorized users.

F. Salazar-Anton · J. Lindh

Department of Microbiology, Tumor and Cell Biology,

Karolinska Institutet,

P.O. Box 280, SE-171 77, Stockholm, Sweden

F. Salazar-Anton · A. Tellez

Department of Microbiology and Parasitology, Faculty of Medical

Sciences, National Autonomous University of Nicaragua,

León, Nicaragua

\section{A. Tellez}

Infectious Disease Center, Faculty of Medical Science,

National Autonomous University of Nicaragua,

León, Nicaragua

\section{J. Lindh $(\bowtie)$}

Epidemiology and Evaluation,

Swedish Institute for Infectious Disease Control,

SE-171 82, Solna, Sweden

e-mail: johan.lindh@smi.se serology for Chagas diseases. Of the 100 serum samples from epileptic patients, 13 were positive and 87 negative by EITB. Compared to Western blot Tsol-p27, immunodot blot Tsolp27 offered similar specificity (97.8\% vs. 95.6\%) but better sensitivity $(86.7 \%$ vs. $76.4 \%)$. The ELISA was similar to the immunodot blot Tsol-p27 regarding both sensitivity and specificity. Western blot TsolHSP36 provided the lowest sensitivity $(61.9 \%)$ and specificity $(86.1 \%)$. None of the antibodies in the serum samples from the Chagas control groups were recognized by immunodot blot Tsol-p27. Our results indicate that the immunodot blot Tsol-p27 provides good sensitivity and specificity. Furthermore, considering the simplicity and low cost of this test, it might be preferable as a diagnostic method in poorly equipped laboratories in endemic countries.

\section{Introduction}

Cysticercosis is a disease caused by larval stages (metacestodes) of Taenia solium, which is considered a public health problem in many low-income countries in Latin America, Africa, and Asia. Notably, this illness is now also emerging in some high-income nations as the result of travel to or immigration from endemic areas (Mahanty and Garcia 2010; Sciutto et al. 2000). Cysticercosis develops after accidental ingestion of $T$. solium eggs passed in the feces of human hosts harboring the adult stage of the parasite in their intestines. Once inside a human, the oncospheres hatch and penetrate the intestinal wall, and subsequently invade various tissues, where they encyst to form cysticerci (Flisser 1994; Raether and Hanel 2003). The main clinical manifestation of the infection is neurocysticercosis (NCC), which occurs when cysticerci are established in the tissues of the brain and central nervous system (Garcia et al. 2002). The clinical signs and symptoms of NCC are inconsistent and 
nonspecific, and include severe headache, hydrocephalus, blindness, epilepsy, and various neurological symptoms (Del Brutto 2005). NCC is a major cause of adult-onset epilepsy in areas where T. solium is prevalent (Flisser 1994). Moreover, in endemics countries, T. solium has been identified as the primary etiological agent of epileptic seizures, accounting for $30-50 \%$ of all cases of late-onset epilepsy (Lescano et al. 2009).

Diagnosis of NCC is complicated because of the unspecific clinical manifestation of this disease. Accurate diagnosis can be achieved by use of neuroimaging methods such as magnetic resonance imaging (MRI) and computed tomography (CT), followed by a confirmatory serological test (Carpio et al. 1998). Unfortunately, in countries where $T$. solium is endemic, radio-imaging methods are seldom affordable and therefore inaccessible to the population at risk (Diaz et al. 1992). The laboratory diagnosis of cysticercosis is based on serological detection of antibodies against cysticerci (Deckers and Dorny 2010). Enzyme-linked immunoassay (ELISA) and enzyme-linked immunoelectrotransfer blot (EITB) are the most frequently used immunodiagnostic tests for human cysticercosis (Dorny et al. 2003); both those methods are specific and sensitive, but they also have the disadvantage of requiring expensive, specialized equipment that must be run by skilled technicians (Deckers and Dorny 2010; Tsang et al. 1989). Thus, there is a continuing need for alternative diagnostic methods that are practical, reproducible, and economical, and also reduce the use of crude material (Deckers and Dorny 2010; Lundstrom et al. 2010; Salazar-Anton and Lindh 2011). Applying recombinant DNA technology can generate antigenic proteins that can serve as simplified, low-cost substitutes for native antigens. As an example of this, the recombinant form of the Tsol-p27 protein identified in T. solium cysticerus might prove useful as a target for diagnostic tools (Salazar-Anton and Lindh 2011). Our aim here was to compare results obtained with an immunodot blot assay based on the Tsol-p27 protein and different immunodiaganostic formats and antigens, using an EITB that we regarded as the gold standard method (Tsang et al. 1989).

\section{Materials and methods}

Serum sample collection

A total of 100 human serum samples were collected from epileptic patients who attended the health centers of the Departments of Chinandega and Rosita in Nicaragua between March and August 2010.The inclusion criteria for epileptic patients were based on clinical signs, symptoms (including seizure unrelated to withdrawal of drugs or alcohol), physical examination, epidemiologic information, and other associated risk factors.

In addition, 60 serum samples with positive (30 sera) or negative (30 sera) indirect immunofluorescence (IF) serology for Chagas disease were included. All sera were stored at $-20^{\circ} \mathrm{C}$ until used. The study protocol was approved by the Ethics Committee of the Faculty of Medical Science at the National Autonomous University of Nicaragua-León (UNAN-León).

Expression and purification of recombinant Tsol-p27 and TsolHSP36 proteins

The process of cloning the Tsol-p27 and TsolHSP36 antigens has been described previously (Salazar-Anton and Lindh 2011). In short, the genes corresponding to the Tsol-p27 and TsolHSP36 antigens were inserted into the plasmid vector pGEX-4T-1 (Amersham Pharmacia) downstream of glutathione $S$-transferase (GST). Both recombinant proteins were expressed in Escherichia coli BL21 (Invitrogen) and purified. In addition to the purification step with glutathione-agarose beads, Tsol-p27 antigen was further purified using his-selected nickel magnetic beads (Sigma). The resin was equilibrated with a buffer containing $50 \mathrm{mM}$ sodium phosphate ( $\mathrm{pH} \mathrm{8.0)}$, $0.3 \mathrm{M}$ sodium chloride, and $10 \mathrm{mM}$ imidazole, and then eluted with a higher concentration of imidazole $(250 \mathrm{mM})$. The histidine-containing proteins were separated from the fraction by applying a magnetic separator to the mixed solution. The antigen purity was analyzed by $10 \%$ SDS PAGE and visualized by staining with Coomassie Brilliant Blue.

\section{EITB (commercial)}

EITB was performed according to the procedure recommended by the manufacturer (LDBIO Diagnostic, Lyon, France).

\section{Antibody-detection ELISA (commercial)}

A commercial ELISA was used according to the manufacturer's instructions (Cypress Diagnostics). This test detected the presence of IgG antibodies against $T$. solium cysticerci in serum samples. A spectrophotometer $(450 \mathrm{~nm})$ was used to obtain optical density reading. Various controls were used for the validity of the ELISA including antibody blank, negative control serum, and positive control serum. The reaction threshold was 0.600 . Values $\geq 0.600$ were considered positive for cysticercosis.

Western blot analysis of Tsol-p27 and TsolHSP36 antigens (in house)

The recombinant antigens were separated by $10 \%$ sodium dodecyl sulfate polyacrylamide gel electrophoresis (SDSPAGE) and transferred onto nitrocellulose membranes (GE 
Healthcare). A positive sample was recorded if a band could be seen at $27 \mathrm{kDA}$ in the Western blot Tsol-p27 and at $36 \mathrm{kDa}$ in the Western blot TsolHSP36. The membranes were blocked for $2 \mathrm{~h}$ with $5 \%$ skim milk in phosphatebuffered saline (PBS, $\mathrm{pH} 7.5$ ) and then rinsed in washing buffer containing PBS and $0.05 \%$ Tween 20 . Thereafter, the membranes were incubated first for $3 \mathrm{~h}$ with human sera diluted 1:500 in 5\% skim milk-PBS, and then for $1 \mathrm{~h}$ with rabbit anti-human IgG conjugated with peroxidase (Sigma) diluted 1:3000 in 5\% skim milk-PBS. The subsequent washing steps and detection procedures were performed according to the ECL Plus manual (GE Healthcare).

Immunodot blot analysis of Tsol-p27 (in house)

Immunodot blot analysis was carried out using a nitrocellulose membrane (GE Healthcare), which was pre-wetted with distilled water and then placed into a bio-dot blot apparatus (BioRad, Hercules, CA, USA). This was followed by fixation of $10 \mu \mathrm{g}$ of recombinant Tsol-p 27 protein that had been purified using glutathione agarose and his-selected nickel beads. The apparatus was left at room temperature for $1 \mathrm{~h}$ to allow antigen immobilization. The dots were subsequently blocked for $1 \mathrm{~h}$ with 5\% skim milk in PBS containing 0.05\% Tween 20 and then washed three times with PBS containing 0.05\% Tween 20 . Thereafter, the dots were incubated for $4 \mathrm{~h}$ with human sera diluted 1:1000 in 5\% skim milk-PBS and washed three times with PBS containing $0.05 \%$ Tween 20 , and this was followed by addition of $100 \mu$ l of rabbit anti-human IgG conjugated with peroxidase (Sigma) diluted 1:4000 in 5\% skim milk-PBS. The subsequent washing steps and detection procedures were performed according to the ECL Plus manual (GE Healthcare). All analyses of the serum samples were performed in duplicate.

\section{Results}

To increase the efficacy and reduce the background of the in house immunodot blot method, the Tsol-p27 protein was purified using his-selected nickel magnetic agarose and glutathione-agarose beads. Figure 1 shows the Tsol-p27 antigen in a $10 \%$ SDS-PAGE, visualized by staining with Coomassie Brilliant Blue.

The sensitivity and specificity values were determined based on the positive and negative results for Tsol-p27 antigen obtained by commercial EITB analysis, the gold standard method. Of the 100 investigated serum samples from epileptic patients, 13 were positive and 87 negative by EITB. The same number of serum samples was analyzed using immunodot blot Tsol-p27, and an example of the results for sera from epileptic patients and patients with positive and negative serology to Chagas diseases is presented in Fig. 2. Corresponding results obtained by the

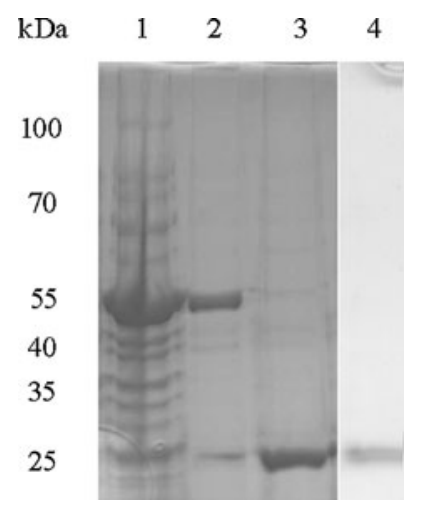

Fig. 1 SDS-PAGE analysis of Tsol-p27 antigen. Lane 1, cells from induced E. coli; lane 2, purified recombinant GST/Tsol-p27; lane 3, Tsol-p27 digested with precision protease; lane 4, Tsol-p27 after purification using glutathione-agarose and his-selected nickel magnetic beads

commercial ELISA and in house Western blot Tsol-p27/ TsolHSP36 are shown (Tables S1 and S2 in the Electronic supplementary material). Table 1 summarizes the performance of the different immunodiagnostic tests compared to the gold standard method EITB for detection of antibodies in serum from epileptic human subjects.

The immunodot blot Tsol-p27 was found to offer $86.7 \%$ sensitivity and $97.8 \%$ specificity, with a positive predictive value (PPV) of $86.7 \%$ and a negative predictive value (NPV) of 97.8\%. Corresponding values for the other tests were as follows: ELISA, 86.7\% sensitivity and 94.6\% specificity (PPV 72.2\%, NPV 97.8\%); Western blot Tsol-p27, 76.4\% sensitivity and 95.6\% specificity (PPV 76.5\%, NPV 98.9\%); Western blot TsolHSP36, 61.9\% sensitivity and 86.1\% specificity (PPV 48.1\%, NPV 91.5\%, Table 2).

\section{Discussion}

Immunodiagnosis of human cysticercosis has been accomplished using a variety of methods, including indirect hemagglutination tests, ELISA, indirect fluorescent antibody tests, radioimmunoassay, complement fixation assays, immunoelectrophoresis, dot blot, and EITB (Deckers and

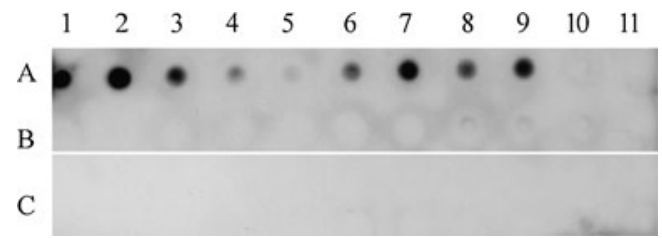

Fig. 2 Immunodot blot using the Tsol-p27 antigen. a Probing done with two positive serum controls $(A 1-A 2)$ followed by sera from seven positive epileptic cases $(A 3-A 9)$ and two negative serum controls (A10-A11). b, c Probing done with negative serum samples from epileptic cases (b), and with sera from patients with positive (1-6) or negative (7-11) serology for Chagas disease (c) 
Table 1 Comparison of different immunodiagnostic tests with EITB as the gold standard method for detection of antibodies against $T$. solium cysticerci in serum samples from epileptic patients

\begin{tabular}{|c|c|c|c|}
\hline & \multicolumn{3}{|l|}{ EITB } \\
\hline & Positive & Negative & Total \\
\hline \multicolumn{4}{|c|}{ Immunodot blot Tsol-p27 } \\
\hline Positive & 13 & 5 & 18 \\
\hline Negative & 2 & 87 & 89 \\
\hline Total & 15 & 92 & 102 \\
\hline \multicolumn{4}{|c|}{ Commercial ELISA } \\
\hline Positive & 13 & 5 & 18 \\
\hline Negative & 2 & 87 & 89 \\
\hline Total & 15 & 92 & 102 \\
\hline \multicolumn{4}{|c|}{ Western blot Tsol-p27 } \\
\hline Positive & 13 & 4 & 17 \\
\hline Negative & 1 & 87 & 88 \\
\hline Total & 14 & 91 & 105 \\
\hline \multicolumn{4}{|c|}{ Western blot TsolHSP36 } \\
\hline Positive & 13 & 14 & 27 \\
\hline Negative & 8 & 87 & 95 \\
\hline Total & 21 & 101 & 122 \\
\hline
\end{tabular}

Dorny 2010; Garcia et al. 2005; Mandal et al. 2008; Simac et al. 1995). The most specific test developed thus far is EITB, with reported specificity of $100 \%$ and sensitivity of $98 \%$. However, the sensitivity of this test drops considerably in cases involving single cysts in the brain (Singh et al. 1999). The EITB method uses an enriched fraction of glycoproteins prepared by lectin-affinity purification from an extract of intact cysticerci (Deckers and Dorny 2010; Tsang et al. 1989). Some of the drawbacks of EITB are that it requires sophisticated equipment and trained personnel to perform the test, it is not suitable for field studies, and it is seldom affordable in countries where cysticercosis is endemic (Deckers and Dorny 2010; Dorny et al. 2003). Therefore, research projects have been focused on developing new serological diagnostic tools that offer sensitivity and specificity, and are easy to perform in poorly equipped laboratories. The aim of the present study was to study the efficacy of an immunodot blot using the Tsol-p27 protein (immunodot blot Tsol-p27) in comparison with various immunodiganostic formats and antigens. More specifically, a commercial ELISA and the antigens TsolHSP36 and Tsol- p27 in Western blot formats (Western blot Tsol-p27/ TsolHSP36) were included in the serological evaluation.

The Tsol-p27 antigen was efficiently expressed in E. coli as a fusion protein with his and GST fusion parts, and it was purified in two steps using glutathione-agarose and hisselected nickel beads. Our immunodot blot technique used a highly purified Tsol-p27 antigen, which decreased the background (e.g., E. coli antigen) seen in Western blot analysis using one-step purified Tsol-p27 antigen (Fig. 1, lane 3). The EITB results were considered confirmatory of human cysticercosis and were used as a standard to assess the performance of the different diagnostic methods. A total of 100 human serum samples from epileptic patients were examined by EITB, and antibodies to T. solium cysticercus were detected in 13 of those samples.

In our study, immunodot blot Tsol-p27 was comparable to Western blot Tsol-p27 with regard to specificity $(97.8 \%$ vs. $95.6 \%)$ but not sensitivity $(86.7 \%$ vs. $76.4 \%)$, and it was similar to ELISA regarding both specificity $(97.8 \%$ vs. $94.6 \%)$ and sensitivity $(86.7 \%$ vs. $86.7 \%)$. Western blotTsolHSP36 showed lower specificity (86.1\%) and sensitivity (61.9\%) compared to the other immunodiagnostic methods. It has previously been noted that the Tsol-p27 antigen has potential for diagnosis of human cysticercosis. In an earlier study (Salazar-Anton and Lindh 2011), Western blot showed that antibodies in serum from NCC patients, but not those in serum from a control group, could identify the Tsol-p27 antigen. Furthermore, it has been reported that using ELISA to detect specific antibodies against $T$. solium cysticerci in samples from patients with suspected cysticercosis provides a useful tool for diagnosing this disease in humans (Rosas et al. 1986; Simac et al. 1995). However, the results of ELISA used for this purpose vary significantly (Bucardo et al. 2005; Carpio 1998), and the discrepancies between studies can be explained by the use of disparate criteria for patient selection and different methods as the gold standard. In our investigation, Western blot TsolHSP36 offered lower sensitivity and specificity compared to immunodot blot Tsolp-27, Western blot Tsolp27, and the commercial ELISA. Notably, the TsolHSP36 antigen has previously been described as an antigenic target in the diagnosis of human cysticercosis (Ferrer et al. 2005).

We also used immunodot blot Tsol p-27 to analyze serum samples from patients with positive or negative serology for Chagas disease, and none of those sera recognized the Tsolp27 antigen. There is an overlap in the epidemiology of
Table 2 Sensitivity, specificity, and PPV and NPV values of the immunodiagnostic tests used to diagnose $T$. solium cysticerci in serum samples from epileptic patients

\begin{tabular}{lcccc}
\hline Immunodiagnostic test & Sensitivity & Specificity & PPV & NPV \\
\hline Immunodot blot Tsol-p27 & $86.7 \%$ & $97.8 \%$ & $86.7 \%$ & $97.8 \%$ \\
Commercial ELISA & $86.7 \%$ & $94.6 \%$ & $72.2 \%$ & $97.8 \%$ \\
Western blot Tsol-p27 & $76.4 \%$ & $95.6 \%$ & $76.5 \%$ & $98.9 \%$ \\
Western blot TsolHSP36 & $61.9 \%$ & $86.1 \%$ & $48.1 \%$ & $91.5 \%$ \\
\hline
\end{tabular}


Chagas disease and human cysticercosis, both of which are considered to be neglected tropical illnesses and occur in poor rural communities of low-income countries in Latin America (Bethony et al. 2011).

At present, T. solium antigens for diagnosis of human cysticercosis are derived from parasites extracted from infected pork (Deckers and Dorny 2010). Perhaps recombinant antigens can serve as a simplified, low-cost substitute for the native antigens, and also provide a reproducible, field-applicable diagnostic assay (Scheel et al. 2005). The immunodot blot Tsol-p27 test showed good sensitivity and specificity in our study, and it is simpler to perform and less expensive than ELISA, EITB, and Western blot. Moreover, immunodot blot Tsol-p27 does not require a spectrophotometer to read the results or electrophoresis and transfer equipment to absorb the antigen onto nitrocellulose membranes (Plancarte et al. 1994). It is plausible that this test will prove to be the serodiagnostic method of choice for use in the field or in poorly equipped laboratories in endemic countries.

Acknowledgments We thank the Swedish International Development Cooperation Agency for providing support via the Infectious Diseases Program, Parasitic Infections, at UNAN-León. We are also thankful to Rosario Palma for helping us in serum sample collection. The study protocol was approved by the Ethics Committee of the Faculty of Medical Science at the National Autonomous University of Nicaragua-León (UNAN-León).

Conflict of interest The authors declare that they have no conflict of interest.

Open Access This article is distributed under the terms of the Creative Commons Attribution Noncommercial License which permits any noncommercial use, distribution, and reproduction in any medium, provided the original author(s) and source are credited.

\section{References}

Bethony JM et al (2011) Vaccines to combat the neglected tropical diseases. Immunol Rev 239(1):237-270. doi:10.1111/j.1600065X.2010.00976.X

Bucardo F, Meza-Lucas A, Espinoza F, Garcia-Jeronimo RC, GarciaRodea R, Correa D (2005) The seroprevalence of Taenia solium cysticercosis among epileptic patients in Leon, Nicaragua, as evaluated by ELISA and western blotting. Ann Trop Med Parasitol 99(1):41-45. doi:10.1179/136485905X19856

Carpio A (1998) Diagnostic criteria for human cysticercosis. J Neurol Sci 161(2):185-188. doi:S0022510X98002275

Carpio A, Escobar A, Hauser WA (1998) Cysticercosis and epilepsy: a critical review. Epilepsia 39(10):1025-1040

Deckers N, Dorny P (2010) Immunodiagnosis of Taenia solium taeniosis/ cysticercosis. Trends Parasitol 26(3):137-144. doi:10.1016/j. pt.2009.12.008

Del Brutto OH (2005) Neurocysticercosis. Semin Neurol 25(3):243251. doi:10.1055/s-2005-917661

Diaz JF et al (1992) Immunodiagnosis of human cysticercosis (Taenia solium): a field comparison of an antibody-enzyme-linked immunosorbent assay (ELISA), an antigen-ELISA, and an enzymelinked immunoelectrotransfer blot (EITB) assay in Peru. The Cysticercosis Working Group in Peru (CWG). Am J Trop Med Hyg 46 (5):610-615

Dorny P, Brandt J, Zoli A, Geerts S (2003) Immunodiagnostic tools for human and porcine cysticercosis. Acta Trop 87(1):79-86. doi: S0001706X03000585

Ferrer E et al (2005) Taenia solium: characterization of a small heat shock protein (Tsol-sHSP35.6) and its possible relevance to the diagnosis and pathogenesis of neurocysticercosis. Exp Parasitol 110(1):1-11

Flisser A (1994) Taeniasis and cysticercosis due to Taenia solium. Prog Clin Parasitol 4:77-116

Garcia HH et al (2002) Current consensus guidelines for treatment of neurocysticercosis. Clin Microbiol Rev 15(4):747-756

Garcia HH, Del Brutto OH, Nash TE, White AC Jr, Tsang VC, Gilman RH (2005) New concepts in the diagnosis and management of neurocysticercosis (Taenia solium). Am J Trop Med Hyg 72(1):3-9

Lescano AG et al (2009) Taenia solium cysticercosis hotspots surrounding tapeworm carriers: clustering on human seroprevalence but not on seizures. PLoS Negl Trop Dis 3(1):e371. doi:10.1371/ journal.pntd.0000371

Lundstrom J, Salazar-Anton F, Sherwood E, Andersson B, Lindh J (2010) Analyses of an expressed sequence tag library from Taenia solium, Cysticerca. PLoS Negl Trop Dis 4(12):e919. doi:10.1371/ journal.pntd.0000919

Mahanty S, Garcia HH (2010) Cysticercosis and neurocysticercosis as pathogens affecting the nervous system. Prog Neurobiol 91 (2):172-184. doi:10.1016/j.pneurobio.2009.12.008

Mandal J, Singhi PD, Khandelwal N, Malla N (2008) Evaluation of lower molecular mass (20-24 kDa) Taenia solium cysticercus antigen fraction by ELISA and dot blot for the serodiagnosis of neurocysticercosis in children. Parasitol Res 102(5):1097-1101. doi:10.1007/s00436-008-0933-4

Plancarte A, Fexas M, Flisser A (1994) Reactivity in ELISA and dot blot of purified GP24, an immunodominant antigen of Taenia solium, for the diagnosis of human neurocysticercosis. Int J Parasitol 24(5):733-738. doi:0020-7519(94)90128-7

Raether W, Hanel H (2003) Epidemiology, clinical manifestations and diagnosis of zoonotic cestode infections: an update. Parasitol Res 91(5):412-438. doi:10.1007/s00436-003-0903-9

Rosas N, Sotelo J, Nieto D (1986) ELISA in the diagnosis of neurocysticercosis. Arch Neurol 43(4):353-356

Salazar-Anton F, Lindh J (2011) Taenia solium: a two-dimensional Western blotting method combined with the use of an ESTlibrary for the identification of immunogenic proteins recognized by sera from neurocysticercosis patients. Exp Parasitol 128 (4):371-376. doi:S0014-4894(11)00159-7

Scheel CM et al (2005) Serodiagnosis of neurocysticercosis using synthetic $8-\mathrm{kD}$ proteins: comparison of assay formats. Am J Trop Med Hyg 73(4):771-776

Sciutto E et al (2000) Taenia solium disease in humans and pigs: an ancient parasitosis disease rooted in developing countries and emerging as a major health problem of global dimensions. Microbes Infect 2(15):1875-1890. doi:S1286457900013368

Simac C, Michel P, Andriantsimahavandy A, Esterre P, Michault A (1995) Use of enzyme-linked immunosorbent assay and enzymelinked immunoelectrotransfer blot for the diagnosis and monitoring of neurocysticercosis. Parasitol Res 81(2):132-136

Singh G, Kaushal V, Ram S, Kaushal RK, Dhanuka AK, Khurana S (1999) Cysticercus immunoblot assay in patients with single, small enhancing lesions and multilesional neurocysticercosis. J Assoc Physicians India 47(5):476-479

Tsang VC, Brand JA, Boyer AE (1989) An enzyme-linked immunoelectrotransfer blot assay and glycoprotein antigens for diagnosing human cysticercosis (Taenia solium). J Infect Dis 159(1):50-59 